\title{
BMJ
}

\section{Effect of preventive ( $\beta$ blocker) treatment, behavioural migraine management, or their combination on outcomes of optimised acute treatment in frequent migraine: randomised controlled trial}

\author{
Kenneth A Holroyd, distinguished professor, ${ }^{1,2}$ Constance K Cottrell, assistant research professor, ${ }^{1,2}$ Francis \\ O'Donnell, clinical assistant professor, ${ }^{2,3,4}$ Gary E Cordingley, associate professor, ${ }^{4}$ Jana B Drew, assistant \\ research professor, ${ }^{1,2}$ Bruce W Carlson, associate professor, ${ }^{1}$ Lina Himawan, biostatistician ${ }^{1}$
}

Ohio University, Athens, $\mathrm{OH}$ 45701, USA

${ }^{2}$ Headache Treatment and Research, Westerville, $\mathrm{OH} 43081$, USA

${ }^{3}$ OrthoNeuro Inc, Westerville, $\mathrm{OH}$ 43081

${ }^{4}$ Ohio University College of Osteopathic Medicine, Athens, $\mathrm{OH}$ 45701

Correspondence to: $\mathrm{K}$ A Holroyd, 225 Porter Hall, Athens, $\mathrm{OH}$ 45701, USA holroyd@ohio.edu

Cite this as: BMJ 2010;341:c4871 doi:10.1136/bmj.c4871

\begin{abstract}
Objective To determine if the addition of preventive drug treatment ( $\beta$ blocker), brief behavioural migraine management, or their combination improves the outcome of optimised acute treatment in the management of frequent migraine.

Design Randomised placebo controlled trial over 16 months from July 2001 to November 2005.

Setting Two outpatient sites in Ohio, USA.

Participants 232 adults (mean age 38 years; $79 \%$ female) with diagnosis of migraine with or without aura according to International Headache Society classification of headache disorders criteria, who recorded at least three migraines with disability per 30 days (mean 5.5 migraines/30 days), during an optimised run-in of acute treatment.

Interventions Addition of one of four preventive treatments to optimised acute treatment: $\beta$ blocker $(n=53)$, matched placebo $(n=55)$, behavioural migraine management plus placebo $(n=55)$, or behavioural migraine management plus $\beta$ blocker $(n=69)$.
\end{abstract}

Main outcome measure The primary outcome was change in migraines/30 days; secondary outcomes included change in migraine days/30 days and change in migraine specific quality of life scores.

Results Mixed model analysis showed statistically significant $(P \leq 0.05)$ differences in outcomes among the four added treatments for both the primary outcome (migraines/30 days) and the two secondary outcomes (change in migraine days/30 days and change in migraine specific quality of life scores). The addition of combined $\beta$ blocker and behavioural migraine management $(-3.3$ migraines/30 days, $95 \%$ confidence interval -3.2 to $-3.5)$, but not the addition of $\beta$ blocker alone $(-2.1$ migraines/30 days, -1.9 to -2.2 ) or behavioural migraine management alone ( -2.2 migraines migraines/30 days, -2.0 to -2.4$)$, improved outcomes compared with optimised acute treatment alone ( -2.1 migraines/ 30 days, -1.9 to -2.2$)$. For a clinically significant $(\geq 50 \%$ reduction) in migraines/30 days, the number needed to treat for optimised acute treatment plus combined $\beta$ blocker and behavioural migraine management was 3.1 compared with optimised acute treatment alone, 2.6 compared with optimised acute treatment plus $\beta$ blocker, and 3.1 compared with optimised acute treatment plus behavioural migraine management. Results were consistent for the two secondary outcomes, and at both month 10 (the primary endpoint) and month 16.

Conclusion The addition of combined $\beta$ blocker plus behavioural migraine management, but not the addition of $\beta$ blocker alone or behavioural migraine management alone, improved outcomes of optimised acute treatment. Combined $\beta$ blocker treatment and behavioural migraine management may improve outcomes in the treatment of frequent migraine.

Trial registration Clinical trials NCT00910689.

\section{INTRODUCTION}

Migraine is common, affecting approximately $14 \%$ of women and $6 \%$ of men worldwide ${ }^{12}$; around a third $(31 \%)$ of these people report frequent migraines defined as three or more attacks a month. ${ }^{23}$ Migraine causes severe impairment or bed rest in more than half (57\%) of affected people, markedly impairs quality of life both during and between attacks, increases absenteeism and reduces productivity at work, and is associated with increased healthcare costs. ${ }^{13-7}$ Frequent migraine is also a risk factor for progression from episodic migraine to chronic migraine (at least 15 headache days a month ${ }^{8}$ ), which commonly presents as debilitating daily or near daily headaches. ${ }^{9-11}$ Recent findings suggest that frequent migraines are associated with structural changes in the brain. ${ }^{12-14}$ The personal, family, and societal impact of frequent migraines, plus the high risk of disease progression and possible neurodegenerative changes in the brain, have led to calls for more aggressive management of frequent migraine in primary care. ${ }^{715}$ 
Although frequent migraine is most often treated in primary care, management seems to be problematic. $^{3716-19}$ This may reflect the fact that the management of frequent migraine can be challenging in primary care, needing drugs for acute treatment and for prevention, as well as changes in patients' behaviour. ${ }^{320-24}$

The average reduction in migraines of $40-50 \%$ seen in trials of preventive drug treatment is clinically meaningful but only moderate in magnitude. ${ }^{2125-27}$ Limitations of the evidence base for preventive drugs include the predominance of short term (six months or less) trials, dropout rates that approach $50 \%$ of randomised participants at the primary end point, ${ }^{28-30}$ and the lack of trials comparing different preventive treatments. Behavioural migraine management uses relaxation, thermal biofeedback, stress management, and other behavioural interventions to teach skills for regulating physiological, mental, and behavioural responses to prevent, abort, and cope with migraines. ${ }^{31-33}$ The average reduction in migraines of $40-50 \%$ seen in trials of behavioural migraine management closely parallels results obtained in trials of preventive drugs. ${ }^{3234-36}$ Limitations of the evidence base for behavioural treatments include the predominance of small trials, the few placebo controlled trials, and the lack of trials that compare behavioural interventions with preventive drugs. ${ }^{3435}$

The possibility that the combination of preventive drug treatment and behavioural migraine management can improve on the outcomes achieved with either treatment modality alone is of particular interest but has yet to be formally evaluated. ${ }^{3437}$ Additional information about the comparative benefits of preventive treatment and behavioural migraine management when added to contemporary acute treatment is also needed.

The $5-\mathrm{HT}_{1 \mathrm{~B} / \mathrm{D}}$ agonists or "triptans" have markedly improved the effectiveness of the acute treatment of migraine. ${ }^{3839}$ However, the very effectiveness of contemporary acute treatment creates uncertainty about the benefits of preventive treatment beyond "optimised" acute treatment alone. ${ }^{4041}$ Contemporary acute treatment effectively controls even frequent migraines in some people. ${ }^{4041}$ Administered early in the migraine episode, acute treatment can terminate a migraine, ${ }^{42-46}$ preventing some of the associated psychosocial and biological consequences of a full blown migraine episode (Bernstein R, personal communication, 2008). ${ }^{164748}$ On the other hand, the additional benefits of a preventive treatment may be smaller when acute treatment is optimised as recommended in clinical practice than when acute treatment is uncontrolled or suboptimal, which is the situation in the typical trial of preventive treatment. ${ }^{4041}$ Information about the benefits of preventive treatment when acute treatment is optimised as recommended in clinical practice is lacking in trials of preventive drugs.

The Treatment of Severe Migraine trial aimed to determine if the addition of preventive drug treatment, behavioural migraine management, or the combination of the two improves the outcomes of optimised acute treatment. Participants with frequent migraines associated with disability received optimised acute treatment, and if their migraines remained uncontrolled they were randomised to one of four added treatments: preventive ( $\beta$ blocker) treatment, placebo, behavioural migraine management plus placebo, or behavioural migraine management plus preventive treatment. $\mathrm{We}$ obtained electronic headache diary recordings for the full 16 months of the trial, including a 12 month evaluation phase, and we assessed migraine related impairments in quality of life at multiple points over the 16 months of the trial.

\section{METHODS}

\section{Participants}

We recruited participants from physicians' referrals and local advertisements at two outpatient sites: one site with two offices serving greater Columbus, Ohio, and one site in Athens, Ohio, serving rural southeastern Ohio. Inclusion criteria were age 18 to 65 years, diagnosis of migraine (with or without aura) according to the international classification of headache disorders criteria at two separate evaluations during the evaluation clinic visit, ${ }^{49}$ and diary confirmed criteria for severity of migraine during the optimised acute treatment run-in of at least three migraines with disability per 30 days. $^{2}$ Exclusion criteria were diagnosis of probable medication overuse headache according to the international classification of headache disorders criteria, a pain disorder other than migraine as the primary presenting problem, 20 or more days with headache a month, contraindication or sensitivity to any study drug, current use of migraine preventive drugs (with participant's preference or welfare contraindicating withdrawal), current psychological treatment, psychiatric disorder needing immediate or priority treatment, and inability to read and understand the study materials; for women, current or planned breast feeding or pregnancy or unwillingness to use an established contraceptive method were also exclusion criteria. All participants gave written informed consent.

\section{Study design and treatments}

During the five week run-in of optimised acute treatment, all participants who met the inclusion criteria at the evaluation in the clinic received optimised acute treatment as described below. Participants who continued to meet the criteria for severity of migraine on the basis of their handheld electronic diary recordings during the run-in period were then randomised, with stratification by sex and by site, to one of the four added treatments: $\beta$ blocker, matched placebo, behavioural migraine management plus $\beta$ blocker, and behavioural migraine management plus placebo. A statistician not otherwise connected with the study generated the randomisation sequence by computer and supplied it in sealed opaque envelopes. After confirming that the electronic headache diary recordings from the run-in period qualified the participant, the site manager 
revealed the randomisation assignment for each participant by opening the next envelope in the sequence. Participants who did not qualify were offered or referred for continuing treatment.

Each of the four treatment protocols required four monthly visits to the clinic and three telephone contacts during the three month treatment/dose adjustment phase (months 1-4) in which the dose of $\beta$ blocker or placebo was adjusted and behavioural migraine management was administered. Telephone contacts were scheduled at the midpoint between the monthly clinic visits in weeks 3,7 , and 10 . During the 12 month (months 5-16) evaluation phase, clinic visits were scheduled at months $5,7,10,13$, and 16 . The $\beta$ blocker and placebo treatments were administered in a standard double blind fashion. Treatment conditions were blinded only for the preventive drug component and not for the administration of behavioural migraine management. The 16 month trial took place between July 2001 and November 2005.

\section{Optimised acute treatment}

The acute treatment protocol emphasised treatment with a $5-\mathrm{HT}_{1 \mathrm{~B} / \mathrm{D}}$ agonist or triptan. Non-steroidal antiinflammatory (ibuprofen) and anti-emetic (metoclopramide) agents could be added as needed..$^{204350}$ The choice of triptans available at the time of the study design (rizatriptan, sumatriptan), the route(s) of administration of triptan (oral, nasal spray, subcutaneous injection), and the addition of a non-steroidal antiinflammatory or anti-emetic agent were tailored to the participant's preference, treatment history, and response to acute treatment. ${ }^{5152}$ Rescue drugs (such as steroids) could also be prescribed. We used individualised handouts and a phone call at week 3 of the run-in period to help participants to evaluate and optimise their acute treatment (for example, to adjust the dose to minimise side effects). Trials of preventive drugs generally have not specified a protocol for acute treatment or attempted to optimise acute treatment but permitted the use of acute treatments as rescue drugs. In these trials, participants recorded use of rescue drugs, but these data have not been reported in publications. ${ }^{28-30}$

\section{$\beta$ blocker and placebo}

In this double blind protocol, we attempted to maximise the efficacy and tolerability of $\beta$ blocker treatment by using a flexible target dose, by treating participants who were unable to tolerate propranolol hydrochloride with nadolol, and by including a proven intervention to maximise adherence. ${ }^{5354}$ Treatment was started with one capsule $(60 \mathrm{mg}$ long acting propranolol hydrochloride or matched placebo) and increased to three capsules (180 mg or matched placebo) at week 12 as tolerated. Participants who did not tolerate at least two capsules $(120 \mathrm{mg}$ ) of long acting propranolol hydrochloride or matched placebo, and in the judgment of the treating neurologist (FJO, GEC) were unimproved, were switched with blindness maintained to the second drug - nadolol for participants who had been receiving propranolol hydrochloride or matched nadolol placebo for participants who had been receiving propranolol placebo. Participants initially received a single $40 \mathrm{mg}$ capsule of nadolol or matched placebo. The dose was increased at the next visit to two capsules (80 mg) as tolerated. At week 12, the dose was stabilised at the highest tolerated level. In the evaluation phase, we permitted an increase to four capsules of long acting propranolol hydrochloride $(240 \mathrm{mg})$ or matched placebo or three capsules of nadolol (120 mg) or matched placebo. We used instructional handouts and the three monthly phone contacts to identify and rectify problems with adherence (this constituted the adherence intervention). ${ }^{5354}$

\section{Behavioural migraine management}

Behavioural migraine management is structured, manually guided treatment that maximises clinical relevance by teaching multiple migraine management skills and tailoring treatment to the participant's progress, preferences, and migraine characteristics. ${ }^{55}$ Migraine management skills are introduced at each of four clinic visits at which the performance of each migraine management skill can be observed and corrected. However, the learning and application of migraine management skills occurs primarily between clinic visits, guided by the behavioural migraine management workbook and accompanying 10 audio lessons. ${ }^{56}$

The first behavioural migraine management session provides an overview of the pathophysiology of migraine, highlighting the relevance of behavioural migraine management skills, and introduces relaxation (muscle stretching, deep breathing, progressive muscle relaxation, relaxation imagery), emphasising the correct performance of progressive muscle (12 muscle groups) relaxation. ${ }^{57}$ Month 1 homework assignments focus on mastering progressively more rapid relaxation techniques and introduce exercises for identifying "prodromal" migraine warning signs and migraine triggers. Building on previous homework, session 2 focuses on the development of a strategy for managing migraine triggers and on using early warning signs as a cue to use relaxation, trigger management, and, where indicated, acute drugs effectively in managing migraines. Month 2 homework assignments focus on the integration of quick (relaxation by recall, cue controlled relaxation) techniques into the participant's daily routine, ${ }^{57}$ as well as on applying and refining the participant's trigger management strategy and plan for responding to early migraine warning signs. Three options are available in session 3: continue with previously introduced "basic" migraine management skills, introduce cognitive-behavioural stress management if stress is a salient migraine trigger, ${ }^{5558}$ or introduce thermal biofeedback ("handwarming") training with a portable digital home thermal biofeedback device (Model SC-911, Bio-Medical Instruments, Warren, MI) if stress is not a notable migraine trigger. ${ }^{55}$ In each case, the relevant behavioural migraine management skills are introduced and performed by participants in session 3, allowing for corrective feedback, and the behavioural migraine 
management manual and audiotapes guide further learning, application, and refinement of the migraine management skills in month 3. At the final clinic session, problems encountered in using behavioural migraine management skills are resolved and a written migraine management plan is prepared on the basis of participants' preferences and observations of the usefulness of the various migraine management activities attempted during treatment. Lastly, relapse prevention or ways of coping with problems that commonly arise in effectively using behavioural migraine management skills over the long term are tackled. For participants receiving behavioural migraine management, we used the three monthly phone contacts to identify and solve problems encountered in using behavioural migraine management skills as well as to identify and correct any drug adherence problems. A more detailed description of treatment procedures has been given by Lipchik and colleagues. ${ }^{55}$

Three masters level and two doctoral level health psychologists administered behavioural migraine management in four sessions of one hour each at the four monthly clinic visits also used for adjustments of doses of drugs. Behavioural migraine management therapists had training that included study of and examination on elements of the treatment protocol, role playing of each therapy session until competence had been demonstrated, and treatment of training cases that were tape recorded for supervision until competence was demonstrated. All trial sessions were tape recorded and reviewed on an ongoing basis for adherence to the protocol.

\section{Outcome measures}

Participants recorded headaches, associated symptoms, and other characteristics of their headaches, as well as drug use, in a handheld electronic (Palm OS ) diary for the 16 months of the trial..$^{5960}$ The number of migraines per 30 days (with at least a 24 hour pain-free period between distinct migraines) and the number of migraine days (defined as a day, from 0001 to 2400, in which a migraine was recorded) per 30 days came from the electronic diary. We assessed the impact of migraines on quality of life with the migraine specific quality of life version 2.1, a 14 item self reported measure with established psychometric properties. ${ }^{6162}$ The migraine specific quality of life was administered by computer before the run-in of optimised acute treatment, at clinic visits 1 and 3 during the treatment/ dose adjustment phase, as well as at each of the five clinic visits during the evaluation phase. The timeframe for the migraine specific quality of life was one month, so the migraine specific quality of life score and the (monthly) migraine diary data were assessed for the same time period. The total score for migraine specific quality of life ranges from 14 to 84, with higher scores reflecting greater impairment in quality of life.

\section{Hypotheses and statistical methods}

We designed this study to determine if the addition of $\beta$ blocker, behavioural migraine management, or combined $\beta$ blocker plus behavioural migraine management improved outcomes with optimised acute treatment alone and, secondarily, whether the three added treatments differed in effectiveness.

The primary outcome was change in number of migraines per 30 days at month 10 relative to the runin period. Secondary outcomes included change in number of days with migraine per 30 days and change in migraine related impairments in quality of life (migraine specific quality of life scores) at month 10 relative to the run-in period; longer term changes in all three outcome variables at month 16 were secondary outcomes. Finally, we calculated the proportion of participants who showed a clinically significant improvement in migraines, defined as at least a 50\% reduction in migraines per 30 days at month 10 as a secondary outcome. ${ }^{63}$

We used $\chi^{2}$ tests to compare attrition and side effects across the relevant treatment groups. Efficacy analyses were intention to treat analyses that included all randomised participants $(n=232)$. We used a mixed model with fixed effects for treatment (four treatment conditions), time (defined as the natural log of months), and the treatment by time interaction to obtain maximum likelihood estimates of missing values and to evaluate treatment effects (PROC MIXED, SAS 9). ${ }^{64-66}$ A mixed model for repeated measures not only can yield unbiased estimates of parameters when missing observations are explained by observed outcomes, but not by unobserved outcomes (missing at random), but also performs surprisingly well even on simulated clinical trial data that include both observations missing at random and observations missing not at random. ${ }^{67-70}$

We fitted the above model by using the restricted maximum likelihood estimation to obtain the best fitting variance-covariance matrix. We used the best fitting, by the Akaike's information criterion, heterogeneous toeplace covariance structure to account for within participant correlation over time for all outcome analyses. As noted above, we used a mixed model with full maximum likelihood estimation to obtain estimates for the fixed effects for all outcome analyses. We modelled all three outcome variables as continuous variables with normal errors. For migraine specific quality of life scores only, a score for before the run-in of optimised acute treatment was available; we thus included this pre-treatment score as a covariate in the above model for this analysis.

When the omnibus treatment by time interaction was statistically significant $(\mathrm{P}<0.05$, two tailed), we did six pairwise comparisons with Bonferroni correction $(\mathrm{P}<0.0083)$ at month 10 , the primary end point, and, to evaluate longer term treatment effects, at month 16. The first three comparisons considered the primary study question by comparing each of the three additive treatments with optimised acute treatment plus placebo. Three additional comparisons compared the three added treatments with one another. At the primary end point at month 10, we also compared the proportion of participants who showed clinically significant improvements, defined as at least a 50\% 
improvement in migraines per 30 days relative to the run-in of optimised acute treatment, ${ }^{63}$ across the four treatment groups by using $\chi^{2}$, with missing values imputed as described above. If this omnibus $\chi^{2}$ was significant $\left(\mathrm{P}<0.05\right.$, two tailed), we used $\chi^{2}$ tests with Bonferroni correction $(\mathrm{P}<0.0083)$ to test the above six comparisons.

Two sensitivity analyses repeated the above mixed model analyses with only participants $(n=214)$ who provided at least one post-randomisation data point and with only participants $(\mathrm{n}=182)$ who completed the treatment/dose adjustment phase and were assessed at month 5 . The post-tests power to detect a between group difference in improvement of 1 (SD 1.5) migraine per 30 days, an adjusted $\alpha$ of 0.0083 , and our initially planned number of participants of 240 was 0.83 .

\section{RESULTS}

\section{Participants' characteristics}

Table 1 shows the demographic and clinical characteristics of the 232 participants. Participants were predominantly $(79 \%)$ female and, on average, recorded 5.5 migraines and 8.5 days with migraine per 30 days. The previous provider of headache care was a primary care physician or gynaecologist for $156(68 \%)$ of the 229 participants who provided this information and a neurologist for 29 (13\%); 40 (17\%) were lapsed consulters, defined as not having consulted a physician in the previous six months despite persistent headache problems. $^{71}$

\section{Attrition}

Figure 1 shows the flow of participants through the trial. Of 1382 potential participants, 997 were eliminated at the initial phone screening: 555 did not meet the criteria for diagnosis or severity of migraine, 174 reported an exclusionary medical condition or contraindication or intolerance to a study drug, 71 were taking or did not wish to take preventive treatment for migraine, 111 were not interested in participation, and 86 could not be scheduled for evaluation in the clinic (lost to follow-up or lived at too great a distance for clinic visits). At the subsequent evaluation, 76 potential participants were excluded: 33 chose not to participate, and 43 were judged ineligible. Of the 43 participants judged ineligible, nine did not have a primary diagnosis of migraine, four had a diagnosis of probable medication overuse headache, 24 had a contraindication or previous intolerance to a study drug, five had a psychiatric disorder that needed priority treatment, and one had another pain disorder as the primary presenting problem. Finally, 77 potential participants were eliminated at the end of the run-in of optimised acute treatment on the basis of their diary recordings: 44 recorded 20 or more headache days per 30 days, and 33 did not meet the criteria for severity of migraine.

Table 1|Demographic and clinical characteristics. Values are numbers (percentages) unless stated otherwise

Characteristics

Demographics

\begin{tabular}{|c|c|c|c|c|c|}
\hline Mean (SD) age at entry (years) & $39.5(10.2)$ & $37.7(10.1)$ & $37.1(9.4)$ & $38.3(10.9)$ & $38.2(10.2)$ \\
\hline Female sex & $45(82)$ & $45(85)$ & $45(82)$ & $49(71)$ & $184(79)$ \\
\hline \multicolumn{6}{|l|}{ Racial or ethnic group*: } \\
\hline White & $47(85)$ & $44(83)$ & $48(87)$ & $55(80)$ & $194(84)$ \\
\hline Black & 7 (13) & $9(17)$ & 7 (13) & $8(12)$ & $31(13)$ \\
\hline Hispanic & $1(0.2)$ & 0 & 0 & $2(3)$ & $3(1)$ \\
\hline Asian & 0 & 0 & 0 & $2(3)$ & $2(1)$ \\
\hline Pacific Islander & 0 & 0 & 0 & $1(1)$ & $1(0)$ \\
\hline American Indian & 0 & 0 & 0 & $1(1)$ & $1(0)$ \\
\hline Income levelt: & $(n=53)$ & $(n=44)$ & $(n=50)$ & $(n=63)$ & $(n=210)$ \\
\hline$\leq \$ 40000$ & $18(34)$ & $17(39)$ & $19(38)$ & $21(33)$ & $75(36)$ \\
\hline$\$ 40001-80000$ & $22(42)$ & $22(50)$ & $13(26)$ & $25(40)$ & $82(39)$ \\
\hline $1 \$ 80000$ & $13(25)$ & $5(11)$ & $18(36)$ & $17(27)$ & $53(25)$ \\
\hline \multicolumn{6}{|l|}{ Migraine characteristics } \\
\hline Mean (SD) migraines/30 days & $5.5(1.9)$ & $5.2(1.9)$ & $5.6(2.0)$ & $5.7(1.9)$ & $5.5(1.9)$ \\
\hline Mean (SD) migraine days / 30 days & $8.4(3.5)$ & $8.6(3.3)$ & $8.1(3.4)$ & $8.7(4.0)$ & $8.5(3.6)$ \\
\hline Mean (SD) migraine specific quality of life score & $40.3(13.4)$ & $40.3(13.4)$ & $38.5(12.4)$ & $39.4(11.9)$ & $39.6(12.7)$ \\
\hline Migraine with aura & $16(6.9)$ & $10(4.3)$ & $9(3.9)$ & $17(7.3)$ & $52(22)$ \\
\hline \multicolumn{6}{|l|}{ Mean (SD) disease duration (years): } \\
\hline At current frequency & $6.6(8.6)$ & $3.9(4.6)$ & $5.6(6.9)$ & $6.5(8.2)$ & $5.7(7.3)$ \\
\hline Problem headaches & $15.6(10.8)$ & $13.3(9.5)$ & $16(8.7)$ & $15.1(10.0)$ & $15.0(9.8)$ \\
\hline
\end{tabular}

*Self identified.

†22 participants declined to report family income.

Plus placebo $(n=55) \quad$ Plus $\beta$ blocker $(n=69)$

Total $(n=232)$
Optimised acute treatment

Optimised acute treatment plus behavioural migraine management

Plus placebo $(n=55) \quad$ Plus $\beta$ blocker $(n=53)$ 
Figure 1 shows that attrition from the 232 randomised participants was $24 \%$ (56 participants) at month 5, 35\% (81 participants) at month 10, and 49\% (114 participants) over the full 16 months of the trial. Attrition did not differ between the four treatment groups at the month 5,10 , or 16 month evaluations (all $\mathrm{P}>0.70$ ).
Dosing and protocol adherence

Acute treatment

One hundred and twelve (48\%) of the 232 randomised participants were using a triptan for acute treatment before the run-in of optimised acute treatment. However, study neurologists judged previous acute treatment to be suboptimal for all 232 participants.

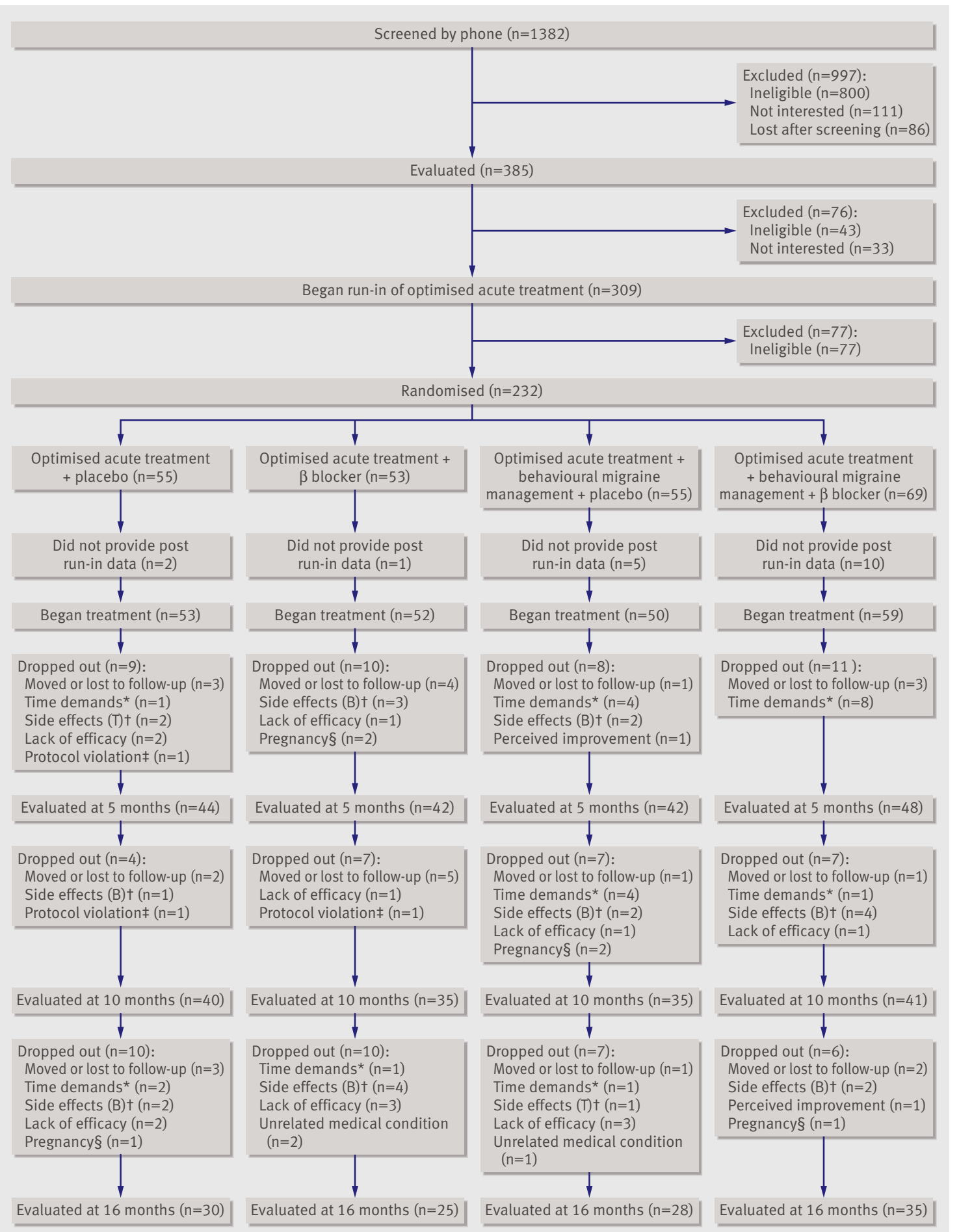

Fig 1 Flow of participants in trial. *Reported that other time demands prevented completion of trial tasks (such as completing study appointments). †Side effects attributed to triptan (T) or $\beta$ blocker (B). $\ddagger$ Discontinued from trial for protocol violation (for example, failed to take preventive drug). §Decided to become or became pregnant 
Triptan use (doses per migraine) did not differ across the four treatment groups, either during the run-in period or at any point in the trial $(\mathrm{P}>0.05)$. All 232 subsequently randomised participants had been treated with a triptan during the run-in of optimised acute treatment, using a mean of 1.2 (SD 0.9) doses per migraine. In month 5, 157/168 (93\%) participants both had a migraine and treated it with a triptan, using a mean of 1.8 (SD 1.1) doses per migraine; in month 10, 124/133 $(93 \%)$ participants both had a migraine and treated it with a triptan, using a mean of 1.8 (1.1) doses per migraine; finally, in month $16,89 / 96$ (93\%) participants both had a migraine and treated it with a triptan, using a mean of 1.9 (1.1) doses per migraine.

\section{Preventive treatment}

At month 5, after dose adjustment, 72/90 (80\%) participants in the active drug groups were taking long acting propranolol hydrochloride (7 at $60 \mathrm{mg}, 18$ at $120 \mathrm{mg}, 47$ at $180 \mathrm{mg}$ ) and 18 were taking nadolol (10 at $40 \mathrm{mg}, 8$ at $80 \mathrm{mg}$ ). At month 10, 61/76 (80\%) participants were taking long acting propranolol hydrochloride $(6$ at $60 \mathrm{mg}, 16$ at $120 \mathrm{mg}, 39$ at $120 \mathrm{mg}, 1$ at $180 \mathrm{mg}$ ) and 15 were taking nadolol (7 at $40 \mathrm{mg}, 6$ at $80 \mathrm{mg}, 2$ at $120 \mathrm{mg}$ ). At month 16, 52/60 $(87 \%)$ participants were taking long acting propranolol hydrochloride (5 at $60 \mathrm{mg}, 14$ at $120 \mathrm{mg}, 33$ at $120 \mathrm{mg}$ ) and eight were taking nadolol ( 5 at $40 \mathrm{mg}, 2$ at $80 \mathrm{mg}, 1$ at $120 \mathrm{mg}$ ).

Daily electronic diary recordings indicated high adherence; about $90 \%$ of participants recorded that they took their preventive drug on at least 25/ 30 days. Specifically, for $\beta$ blocker, 85/90 (94\%) participants at month $5,72 / 76(95 \%)$ participants at month 10 , and 54/59 (92\%) participants with available data at month 16 recorded taking their preventive drug on at least 25/30 days. Resting heart rate was reduced from baseline levels in the $\beta$ blocker groups at each assessment $(-10.6(95 \%$ confidence interval -13.2 to -7.9$)$ beats/min $(\mathrm{n}=89)$ at month $5 ;-9.4(-12.1$ to -6.7$)$ beats/min $(\mathrm{n}=76)$ at month $10 ;-6.8(-9.9$ to -3.6$)$ beats/min $(n=59)$ at month 16$)$, but essentially unchanged in the placebo groups $(-1.1$ ( -3.7 to 1.5$)$ beats/ $\min (\mathrm{n}=85)$ at month $5 ;-0.5(-2.9$ to 1.9$)$ beats/ $\min (\mathrm{n}=74)$ at month $10 ;-1.7(-5.1$ to 1.5$)$ beats/min $(\mathrm{n}=57)$ at month 16).

Adherence to behavioural migraine management was also reasonably high; approximately $70 \%$ of participants showed high adherence to behavioural migraine management homework assignments (defined as completion of at least 60\% of behavioural migraine management homework assignments with good or excellent ratings for quality of homework by the counsellor). Specifically, for the 90 participants receiving behavioural migraine management evaluated at month 5 , adherence data for session 2 were available for 87 , and $62(71 \%)$ of these participants showed high adherence to homework assigned at session 2 ; similarly 68/89 (76\%) participants with available adherence data for session 3 showed high adherence to session 3 homework assignments; finally,
$68 / 88(77 \%)$ participants with available adherence data for session 4 showed high adherence to session 4 homework.

\section{Outcomes}

Mixed model analysis yielded significant effects for both time $(\mathrm{P}<0.001)$ and the treatment by time interaction (at least $\mathrm{P}<0.05$ ) for each of the three outcome measures, indicating statistically significant differences in outcomes with the four treatments over time. At the primary end point of month 10, figures 2 and 3 and table 2 show that each of the four treatments yielded clinically meaningful improvements in number of migraines and days with migraine. However, only the addition of combined $\beta$ blocker and behavioural migraine management improved on the outcomes obtained with optimised acute treatment alone. Differences among the three remaining treatments were neither clinically meaningful in magnitude nor statistically significant. The longer term pattern of results at month 16 was identical.

The proportion of participants classified as clinically improved ( $\geq 50 \%$ reduction in migraines) at the primary end point (month 10) differed similarly across groups $(\mathrm{P}<0.001)$. Optimised acute treatment plus $\beta$ blocker and behavioural migraine management, with 53/69 (77\%) participants clinically improved, differed from the remaining three treatments $(\mathrm{P} \leq 0.001)$, whereas differences among the other three treatments were neither clinically meaningful nor statistically significant: 22/55 (40\%) optimised acute treatment alone participants, 18/53 (34\%) optimised acute treatment plus $\beta$ blocker participants, and 19/55 (36\%) optimised acute treatment plus behavioural migraine management participants were classified as clinically improved. For optimised acute treatment plus $\beta$

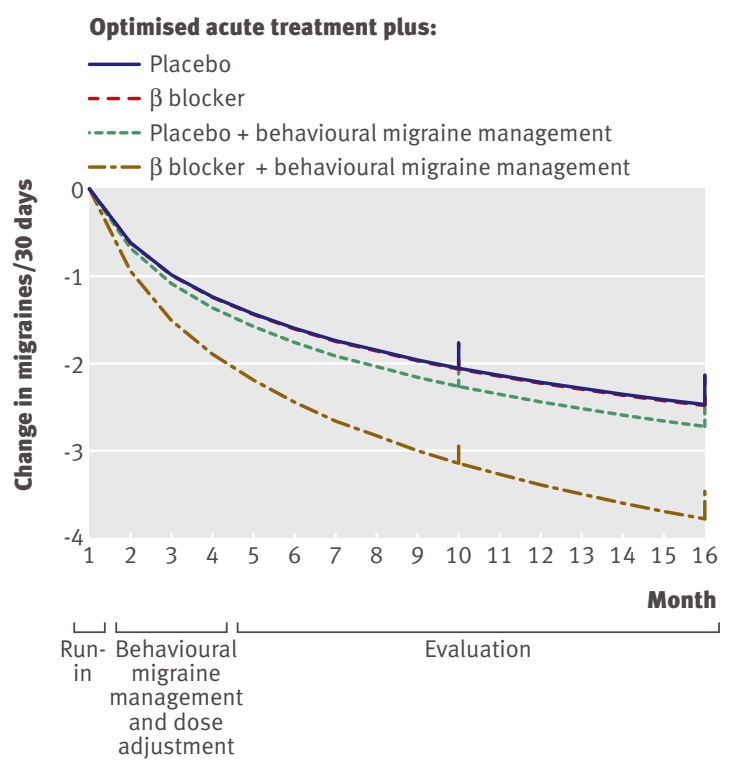

$\overline{\text { Fig } 2}$ Mean change in number of migraines per 30 days from run-in of optimised acute treatment estimated for each of four treatments. Vertical lines at months 10 and 16 indicate primary end point and longer term assessment point 
Table $2 \mid$ Mean change (95\% confidence interval) and results of pairwise comparisons for four treatments at month 10 and month 16

\begin{tabular}{|c|c|c|c|c|}
\hline \multirow[b]{2}{*}{ Outcome measure } & \multicolumn{2}{|c|}{ Optimised acute treatment } & \multicolumn{2}{|c|}{$\begin{array}{l}\text { Optimised acute treatment plus behavioural } \\
\text { migraine management }\end{array}$} \\
\hline & Plus placebo $(n=55)$ & Plus $\beta$ blocker $(n=53)$ & Plus placebo $(n=55)$ & Plus $\beta$ blocker $(n=69)$ \\
\hline \multicolumn{5}{|l|}{ Month 10} \\
\hline Migraines/30 days & $-2.1(-1.9$ to -2.2$)$ & $-2.1(-1.9$ to -2.2$)$ & $-2.2(-2.0$ to -2.4$)$ & $-3.3^{\star}(-3.2$ to -3.5$)$ \\
\hline Migraine days/30 days & $-3.3(-3.0$ to -3.6$)$ & $-3.9(-3.5$ to -4.2$)$ & $-3.3(-2.9$ to -3.7$)$ & $-5.4^{\star}(-5.2$ to -5.6$)$ \\
\hline Migraine specific quality of life scores & $-7.1(-6.3$ to -7.8$)$ & $-7.1(-6.6$ to -7.7$)$ & $-8.6^{*}(-8.2$ to -8.9$)$ & $-13.0^{\star}(-12.5$ to -13.5$)$ \\
\hline \multicolumn{5}{|l|}{ Month 16} \\
\hline Migraines/30 days & $-2.5(-2.3$ to -2.6$)$ & $-2.5(-2.2$ to -2.8$)$ & $-2.7(-2.5$ to -2.9$)$ & $-3.8^{\star}(-3.5$ to -4.0$)$ \\
\hline Migraine days/30 days & $-3.9(-3.5$ to -4.3$)$ & $-4.5(-4.0$ to -5.1$)$ & $-4.1(-3.8$ to -4.5$)$ & $-6.1^{\star}(-5.6$ to -6.6$)$ \\
\hline Migraine specific quality of life scores & $-8.8(-8.1$ to -9.5$)$ & $-8.5(-7.6$ to -9.4$)$ & $-9.6(-9.0$ to -10.3$)$ & $-15.2^{\star}(-14.4$ to -16.0$)$ \\
\hline
\end{tabular}

blocker and behavioural migraine management, the number needed to treat was 3.1 compared with optimised acute treatment alone, 2.6 compared with optimised acute treatment plus $\beta$ blocker, and 3.1 compared with optimised acute treatment plus behavioural migraine management.

Migraine specific quality of life scores were higher at the beginning (mean 39.6, SD 12.7) than at the end of the run-in of optimised acute treatment (mean 34.0, SD 10.3) $(\mathrm{P}<0.01)$. Figure 4 and table 2 show that additional improvements in migraine specific quality of life scores occurred with all four treatments at the primary end point; optimised acute treatment plus $\beta$ blocker and behavioural migraine management yielded improvements that were larger than the remaining three treatments at both the month 10 and month 16 evaluations. Optimised acute treatment plus behavioural migraine management also yielded larger reductions in migraine specific quality of life scores than did optimised acute treatment alone or optimised acute treatment plus $\beta$ blocker at month 10, but differences between optimised

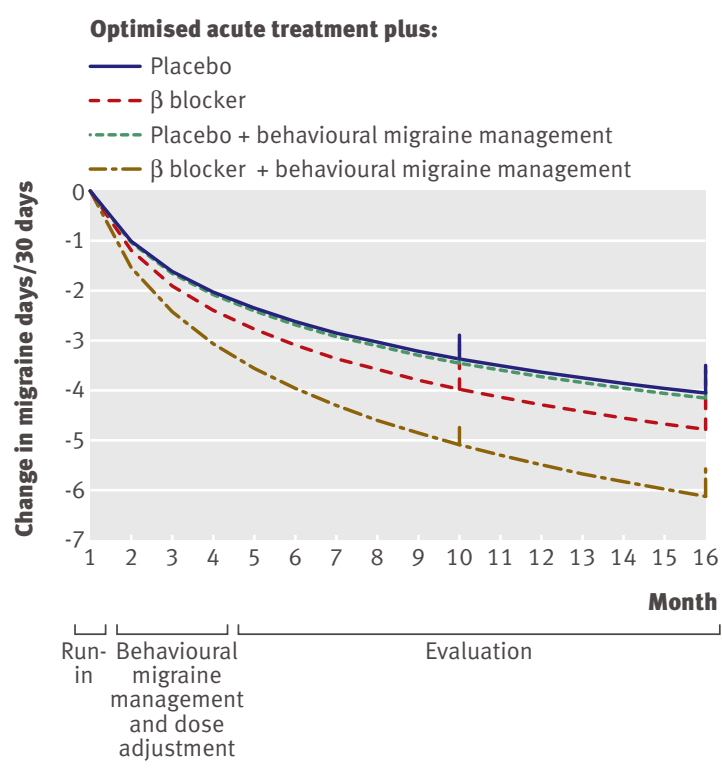

Fig 3 Mean change in number of migraine days per 30 days from run-in of optimised acute treatment estimated for each of four treatments. Vertical lines at months 10 and 16 indicate primary end point and longer term assessment point acute treatment alone and optimised acute treatment plus $\beta$ blocker were not statistically significant at month 16.

The two sensitivity analyses also yielded significant treatment by time interactions $(\mathrm{P} \leq 0.05)$ for each of the three outcome variables. For migraines and for migraine days, the same treatment group differences were statistically significant in the two sensitivity analyses and in the intention to treat analyses at both month 10 and month 16 . For migraine specific quality of life scores, the two sensitivity analyses and the intention to treat analysis differed for two planned comparisons. In the sensitivity analysis $(n=182)$ that included only participants who completed the treatment/dose adjustment phase, differences between optimised acute treatment plus behavioural migraine management and both optimised acute treatment alone and optimised acute treatment plus $\beta$ blocker were statistically significant at both month 10 and month 16, rather than only at month 10 as was seen in the intention to treat analysis.

\section{Adverse effects}

Of the 208 participants who completed the first dose adjustment session, at least one side effect was reported during the dose adjustment phase by 54/110 (49\%) participants receiving $\beta$ blocker and 40/98 (41\%) receiving placebo $(\mathrm{P}=0.23)$. After dose adjustment at month 5 , side effects were reported by only 18/90 (20\%) participants taking $\beta$ blocker and 9/86 (10\%) taking placebo $(\mathrm{P}=0.08)$. The one side effect reported by $5 \%$ or more of participants was fatigue, reported by $9 / 90$ (10\%) participants taking $\beta$ blocker and 5/86 (6\%) taking placebo $(\mathrm{P}=0.31)$. For the 214 participants who provided postbaseline data (fig 1), attrition attributed to the side effects of $\beta$ blocker treatment was 7/110 (6\%) participants assigned to $\beta$ blocker compared with 5/93 (5\%) assigned to placebo $(\mathrm{P}=0.77)$ at month 5 . Over the full 16 months of the trial, attrition attributed to side effects of $\beta$ blocker treatment was 13/101 (13\%) participants assigned to $\beta$ blocker compared with 7/93 (8\%) assigned to placebo $(\mathrm{P}=0.25)$.

\section{DISCUSSION}

To our knowledge, this is the first controlled trial to examine the separate and combined effects of 


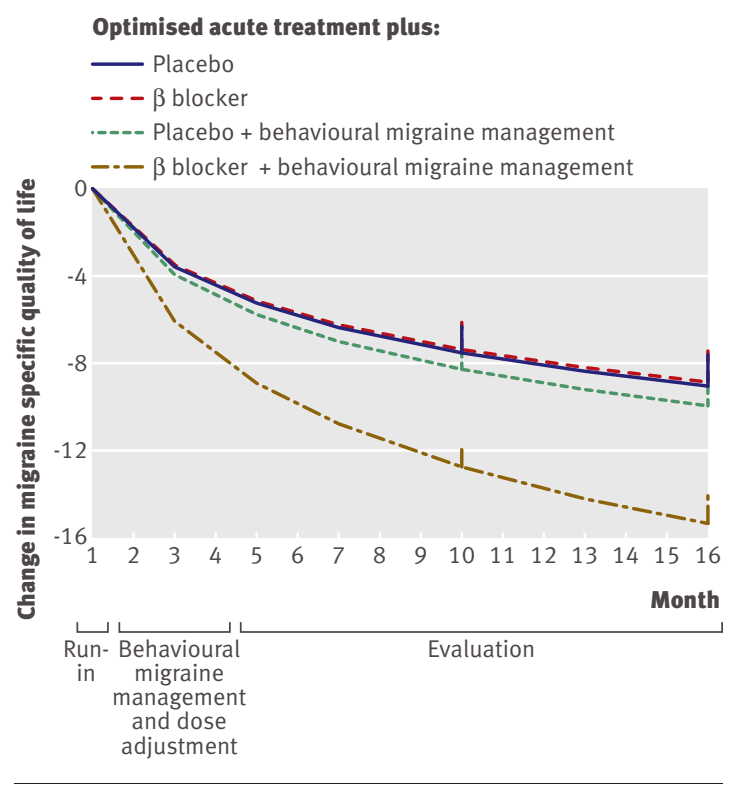

Fig $4 \mid$ Mean change in migraine specific quality of life scores from run-in of optimised acute treatment estimated for each of four treatments. Vertical lines at months 10 and 16 indicate primary end point and longer term assessment point

preventive drug treatment and behavioural migraine management, as well as the first trial to determine if these treatments improve the outcomes obtained with an "optimised" acute treatment regimen. Only the combination of $\beta$ blocker treatment and behavioural migraine management improved outcomes with optimised acute treatment. This combined treatment yielded larger improvements in number of migraines, number of days with migraine, and quality of life at both short term (month 10) and longer term (month 16) assessments than did the other three treatments. Preventive ( $\beta$ blocker) treatment combined with brief behavioural migraine management thus may be a promising method of improving outcomes in the management of frequent impairing episodic migraine.

In contrast to results obtained with combined treatment, neither $\beta$ blocker alone nor behavioural migraine management alone improved on the outcomes of optimised acute treatment. However, neither $\beta$ blocker alone nor behavioural migraine management alone were ineffective. The approximately 50\% reductions in migraine episodes and in migraine days seen with $\beta$ blocker and with behavioural migraine management in this trial compare favourably with the average reductions in migraines seen in previous trials of $\beta$ blocker and of behavioural therapy. ${ }^{215-273234-36}$ However, optimised acute treatment alone yielded improvements in migraines and quality of life of the same magnitude. As a result, we were unable to show that the addition of either $\beta$ blocker alone or behavioural migraine management alone improved on the substantial improvements achieved with optimised acute treatment.

\section{Optimised acute treatment}

The effectiveness of optimised acute treatment in this study is consistent with the observation that the very effectiveness of contemporary acute treatment can limit the benefits obtained with the addition of preventive treatment. ${ }^{4041}$ However, this trial was not designed to evaluate the effectiveness of the optimised acute treatment protocol and thus did not include a comparison group that did not receive either optimised acute treatment or preventive treatment. Concerns about the possible deleterious psychosocial and biological effects of frequent disabling migraines prevented inclusion of this control group. ${ }^{61112147273}$ As a result, we cannot exclude the possibility that improvements seen with optimised acute treatment reflect attention and nonspecific effects associated with participation in a clinical trial, a preventive drug placebo effect, regression to the mean, or the natural course of the disorder. ${ }^{74}$ Participants' longstanding history of frequent migraines and the randomisation of only the participants who had frequent migraines with disability despite receiving optimised acute treatment during the run-in period argue against, but do not eliminate, this possibility. Nevertheless, our results raise the possibility that the results of clinical trials that do not optimise acute treatment will fail to generalise to clinical situations in which acute treatment is optimised, in the manner recommended in current clinical guidelines. ${ }^{43}$ Additional information is thus needed about the benefits clinicians can expect from the addition of preventive drugs to contemporary acute treatment.

Trials of acute treatment may provide only a rough estimate of the benefits of contemporary acute treatment. Trials typically evaluate a single triptan or other agent relative to placebo for, at most, a few migraines. ${ }^{38} 39$ However, contemporary acute treatment involves tailoring the choice of agent (such as triptan) and route(s) of administration and, if needed, adjunctive (for example, anti-emetic or non-steroidal anti-inflammatory) drugs, with the goal of optimising the effectiveness and the tolerability of acute treatment for each patient. ${ }^{75}$ Limitations of insurance coverage or other financial considerations may well constrain clinicians' ability to optimise acute treatment. ${ }^{76}$ However, this type of tailoring of the acute treatment regimen remains the clinical goal. ${ }^{435077}$ Additional information about the effectiveness of optimised acute treatment, as currently recommended, would thus be helpful in evaluating treatment options in frequent migraine.

\section{Limitations of study}

Our findings are necessarily limited to the preventive treatment that we evaluated. However, little evidence indicates that other preventive drugs, including antidepressants and anticonvulsants, are more effective than $\beta$ blockers for episodic migraine, ${ }^{3078-90}$ although adequately powered comparisons of different preventive drugs are unavailable. Similarly, we have little reason to believe that other behavioural interventions are more effective than the behavioural migraine management intervention evaluated here, ${ }^{3234}$ although adequately powered comparisons of different behavioural interventions also are unavailable. Thus, these findings may be generalisable to other preventive drugs and to 


\section{WHAT IS ALREADY KNOWN ON THIS TOPIC}

Frequent migraine has a major impact on quality of life and can be difficult to manage in primary care

Preventive drug treatment and behavioural migraine management each yield moderate improvements in migraine

However, the benefits of these preventive treatments, either alone or when combined, remain unclear when they are administered in the presence of contemporary acute migraine treatment

\section{WHAT THIS STUDY ADDS}

Only the combination of behavioural migraine management and preventive treatment ( $\beta$ blocker) improved outcomes compared with optimised acute treatment alone

other behavioural interventions. However, appropriately designed clinical trials are needed to determine if this is the case.

Strengths of this trial include a relatively low attrition compared with some recent trials, ${ }^{28-30}$ a longer evaluation period, electronic daily diary monitoring of headaches and drug use for the full 16 months of the trial, monitoring of adherence, and relatively high adherence to treatments. ${ }^{3}$ However, we excluded people with a diagnosis of medication overuse headaches or with a comorbid pain disorder as their primary presenting problem in order to evaluate the effect of migraine specific treatments on both migraines and migraine related deficits in quality of life. Our results thus cannot be readily generalised to participants with these disorders as the primary presenting problem. Future studies in primary care settings might examine treatment outcome as a function of comorbid disorders. Also, we cannot exclude the possibility that the inclusion of additional triptans that have become available since this trial was planned would further increase the effectiveness of the optimised acute treatment protocol, although we know of no evidence to indicate that this would be the case.

\section{Conclusions}

Only the combination of $\beta$ blocker treatment and brief behavioural migraine management improved outcomes obtained with optimised acute treatment in this trial. Preventive drugs combined with brief behavioural migraine management may be a promising method for improving treatment outcomes and, possibly, for reducing progression of migraine and neurodegenerative changes seen in the brain in people with frequent migraine.

We thank the following people for their assistance in carrying out the trial: Carol Nogrady, Kimberly Hill, Suzanne Smith, Bernadette Devantes Heckman, Brenda Pinkerman, Gregg Tkachuk, Sharon Waller, Donna Shiels, Kathleen Darchuk, Yi Chen, Timur Skeini, Manish Singla, Swati Dalmai, and Lori Arnott.

Contributors: KAH, CKC, FJO'D, and GEC were responsible for the study concept and design. KAH, BWC, and FJO'D obtained funding. KAH, CKC, FJO'D, GEC, and JBD were involved in acquisition of data. KAH, BWC, and LH analysed and interpreted the data. LH, BWC, and KAH did the statistical analysis. Victor Heh assisted with data analysis. Jenifer Labus did the computerised randomisation. KAH drafted the manuscript, and al authors critically revised it for important intellectual content. KAH, CKC,
FJO'D, and JBD provided administrative, technical, or material support. $\mathrm{KAH}, \mathrm{CKC}$, and JBD supervised the study. $\mathrm{KAH}$ is the guarantor. Funding: Grant R01-NS-32374 (awarded to KAH) from the National Institutes of Health provided primary support for this trial. Merck Pharmaceuticals and GlaxoSmithKline Pharmaceuticals donated triptans for the trial, which was their only involvement. These organisations had no role in the data collection, statistical analysis, or writing of this article or the decision to submit for publication.

Competing interests: KAH has consulted for ENDO Pharmaceuticals and for Takeda Pharmaceuticals North America and received an investigator initiated grant from ENDO Pharmaceuticals. He has also received suppor from the National Institutes of Health (NINDS; NS32375). CKC has received research funding and materials from GlaxoSmithKline Pharmaceuticals (GSK) and Merck and participates in industry sponsored research involving GSK, Merck, UCB Pharma, and Allergan. FJO'D has received research funding and materials from GSK and Merck; receives educational funding from GSK, Merck, and Allergan; participates in industry sponsored research involving GSK, Merck, UCB Pharma, and Allergan; and has consulted for and received honorariums from GSK. GEC owns stock in Johnson and Johnson, Novartis, and Wyeth

Pharmaceuticals.

Ethical approval: All participants provided written informed consent according to procedures approved by the Ohio University Human Subjects Committee.

Data sharing: Technical appendix with statistical code and details of analyses available from the corresponding author at (holroyd@ohio.edu) or biostatistician (himawan@ohio.edu). Consent was not obtained from participants, but risk of identification is low.

1 Stovner LJ, Hagen K, Jensen R, Katsarava Z, Lipton RB, Scher Al, et al. The global burden of headache: a documentation of headache prevalence and disability worldwide. Cephalalgia 2007;27:193-210.

2 Lipton R, Stewart A, Diamond S, Reed M. Prevalence and burden of migraine in the United States: data from the American Migraine Study II. Headache 2001;41:646-57.

3 Lipton R, Bigal M, Diamond M, Freitag F, Reed M, Stewart W. Migraine prevalence, disease burden, and the need for preventive therapy. Neurology 2007;68:343-9.

4 Von Korff M, Stewart W, Simon D, Lipton B. Migraine and reduced work performance-a population-based diary study. Neurology 1998;50:1741-5.

5 Terwindt G, Ferrari M, Tihuis M, Groenen S, Picavet H, Launer L. The impact of migraine on quality of life in the general population: the GEM study. Neurology 2000;55:624-9.

6 Brandes J. The migraine cycle: patient burden of migraine during and between migraine attacks. Headache 2008;48:430-41.

7 Hazard E, Munakata J, Bigal M, Rupnow M, Lipton R. The burden of migraine in the United States: current and emerging perspectives on disease management and economic analysis. Value Health 2009;12:55-64.

8 Olesen J, Bousser M, Diener H, Dodick D, First M, Goadsby P, et al. New appendix criteria open for a broader concept of chronic migraine. Cephalalgia 2006;26:742-6.

9 Silberstein S, Lipton R, Saper J. Chronic daily headache including transformed migraine, chronic tension-type headache and medication overuse headache. In: Silberstein S, Lipton R, Dodick D, eds. Wolff's headache and other head pain. 8th ed. Oxford University Press, 2008:315-78.

10 Bigal M, Lipton R. Concepts and mechanisms of migraine chronification. Headache 2008;48:7-15.

11 Scher A, Midgette L, Lipton R. Risk factors for headache chronification. Headache 2008;48:16-25.

12 Schmitz N, Admiraal-Behloul F, Arkink E, Kruit M, Schoonman G, Ferrari $\mathrm{M}$, et al. Attack frequency and disease duration as indicators for brain damage in migraine. Headache 2008;48:1044-55.

13 Kruit M, Launer L, Overbosch J, van Buchem M, Ferrari M. Iron accumulation in deep brain nuclei in migraine: a population-based magnetic resonance imaging study. Cephalalgia 2008;29:351-9.

14 Aurora S. Is chronic migraine one end of a spectrum of migraine or a separate entity? Cephalalgia 2009;29:597-605.

15 Loder E, Biondi D. General principles of migraine management: the changing role of prevention. Headache 2005;45(suppl 1):33-47S.

16 Harpole L, Samsa G, Matchar D, Silberstein S, Blumenfeld A, Jurgelski A. Burden of illness and satisfaction with care among patients with headache seen in a primary care setting. Headache 2005;45:1048-55.

17 Kernick D. Reducing the burden of headache: the International Headache Society Primary Care Interest Group. Cephalalgia 2010;30:899-901.

18 Cevoli S, Amico DD, Martelletti P, Valguarnera F, Bene ED, Simone RD, et al. Underdiagnosis and undertreatment of migraine in 
Italy: a survey of patients attending for the first time 10 headache centres. Cephalalgia 2009;29:1285-93.

19 Stewart W, Lipton R. The economic and social impact of migraine. Eu Neurol 1994;34(suppl 2):127S.

20 Silberstein S, Freitag F, Bigal M. Migraine treatment. In: Silberstein S, Lipton R, Dodick D, eds. Wolff's headache and other head pain. 8th ed. Oxford University Press, 2008:177-292.

21 Ramadan N, Silberstein S, Freitag F, Gilbert T, Frishberg B. Evidence based guidelines for migraine headache in the primary care setting: pharmacological management for the prevention of migraine. 2002 www.aan.com/professionals/practice/pdfs/gl0090.pdf.

22 Silberstein S. Practice parameter: evidence-based guidelines for migraine headache (an evidence-based review). Neurology 2000;55:754-62.

23 Steiner T, Paemeiere K, Jensen R, Valade D, Savis L, Lainez M, et al. European principles of management of common headache disorders in primary care. J Headache Pain 2007;8(suppl 1):3-47S.

24 Kaniecki RL. Treatment of primary headache: preventive treatment of migraine. In: Freitag F, Cady R, eds. Standards of care for headache diagnosis and treatment. National Headache Foundation, 2004:4052.

25 Holroyd KA, Penzien DD, Cordingley GE. Propranolol in the management of recurrent migraine: a meta-analytic review. Headache 1991;31:333-40

26 Ramadan N, Schultz L, Gilkey S. Migraine prophylactic drugs: proof of efficacy, utilization and cost. Cephalalgia 1997;17:73-80.

27 Rossnagel K. Propranolol for migraine prophylaxis (review). Cochrane Database Syst Rev 2004;2:CD003225.

28 Brandes J, Saper J, Diamond M, Couch J, Lewis D, Schmitt J, et al. Topiramate for migraine prevention. JAMA 2004;291:965-73.

29 Silberstein S, Neto W, Schmitt J, Jacobs D. Topiramate in migraine prevention: results of a large controlled trial. Arch Neurol 2004;61:490-5.

30 Diener H-C, Tfelt-Hansen P, Dahlof C, Lainez M, Sandrini G, Wang S-J, et al. Topiramate in migraine prophylaxis: results from a placebocontrolled trial with propranolol as an active control. J Neurol 2004;251:943-50.

31 Holroyd K, Penzien D, Rains J, Lipchik G, Buse D. Behavioral management of headaches. In: Silberstein S, Lipton R, Dodick D, eds. Wolff's headache and other head pain. 8th ed. Oxford University Press, 2008:721-46.

32 Penzien D, Rains J, Andrasik F. Behavioral management of recurrent headache: three decades of experience and empiricism. Appl Psychophysiol Biofeedback 2002;27:163-81.

33 Holroyd K, Drew J. Behavioral approaches to the treatment of migraine. In: Swanson J, ed. Seminars in neurology: headache. Theme Medical Publishers, 2006:199-207.

34 Campbell J, Penzien D, Wall E. Evidence-based guidelines for migraine headache: behavioral and physical treatments. 2000 www.aan.com/professionals/practice/pdfs/gl0089.pdf.

35 Holroyd K. Assessment and psychological treatment of recurrent headache disorders. J Consult Clin Psychol 2002;70:656-77.

36 Holroyd K, Penzien D. Pharmacological vs nonpharmacological prophylaxis of recurrent migraine headache: a meta-analytic review of clinical trials. Pain 1990;42:1-13.

37 Holroyd K, France J, Cordingley G, Rokicki L, Kvaal S, Lipchik G, et al. Enhancing the effectiveness of relaxation/thermal biofeedback training with propranolol HCl. J Consult Clin Psychol 1995;63:327-30.

38 Saxena P, Tfelt-Hansen $\mathrm{P}$. Triptans, $5-\mathrm{HT}_{1 \mathrm{~B} / 1 \mathrm{D}}$ receptor agonists in the acute treatment of migraines. In: Olesen J, Goadsby P, Ramadan N, Tfelt-Hansen P, Welch K, eds. The headaches. 3rd ed. Lippincott Williams \& Wilkins, 2006:469-504.

39 Ferrari M, Roon K, Lipton R, Goadsby P. Oral triptans (serotonin 5-HT (1B/1D) agonists) in acute migraine treatment: a meta-analysis of 53 trials. Lancet 2001;358:1668-75.

40 Adelman J, Brod A, Von Seggem R, Mannix L, Rappoport A. Migraine preventive medications: a reappraisal. Cephalalgia 1998;18:605-11.

41 Adelman J, Adelman L, Von Seggem R. Cost-effectiveness of antiepileptic drugs in migraine prophylaxis. Headache 2002;42:978-83.

42 Lipton R, Stewart W, Stone A, Lainez M, Sawyer J. Stratified care vs step care strategies for migraine: the Disability in Strategies of Care (DISC) study: a randomized trial. JAMA 2000;284:2599-605.

43 Diamond R, Cady R. Initiating and optimizing acute therapy for migraine: the role of patient-centered stratified care. Am J Med 2005;118(suppl 1):185-275S.

44 Diener H, Ferrari M, Mansbach M. Predicting the response of sumatriptan: the sumatriptan naratriptan aggregate patient database. Neurology 2004;63:520-4.

45 Tfelt-Hansen P. Maximum effect of triptans in migraine? A comment. Cephalalgia 2008;28:767-8.

46 Goadsby P, Zanchin G, Geraud G, de Klippel N, Diaz-Insa S, Gobel H. Early vs no-early intervention in acute migraine-'Act when Mild'
(AwM): a double-blind, placebo-controlled trial of almotriptan. Cephalalgia 2008;28:383-91.

47 Levy D, Jakubowski M, Burstein R. Disruption of communication between peripheral and central trigeminovascular neurons mediates the antimigraine action of $5 \mathrm{HT} 1 \mathrm{~B} / 1 \mathrm{D}$ receptor agonists. Proc Natl Acad Sci U S A 2004;101:4274-9.

48 Sakai Y, Dobson M, Diksic M, Aube M, Hamel E. Sumatriptan normalizes the migraine attack-related increase in brain serotonin synthesis. Neurology 2008;70:431-9.

49 Headache Classification Committee of the International Headache Society. Classification and diagnostic criteria for headache disorders, cranial neuralgias and facial pain. Cephalalgia 1988;8(suppl 7):1-96S.

50 Tfelt-Hansen P. Prioritizing acute pharmacotherapy of migraines. In Olesen J, Goadsby P, Ramadan N, Tfelt-Hansen P, Welch K, eds. The headaches. 3rd ed. Lippincott Williams \& Wilkins, 2006:515-8.

51 Silberstein S, Mannix L, Goldstein J, Couch J, Byrd S, Ames M, et al. Multimechanistic (sumatriptan-naproxen) early intervention for the acute treatment of migraine. Neurology 2008;71:114-21.

52 Azzopardi T, Brooks N. Oral metoclopramide as an adjunct to analgesics for the outpatient treatment of acute migraine. Ann Pharmacother 2008;42:397-402.

53 Peveler R, George C, Kinmonth A, Cambell M, Thompson C. Effect of antidepressant drug counselling and information leaflets on adherence to drug treatment in primary care: a randomised controlled trial. BMJ 1999;319:612-5.

54 Holroyd KA, O’Donnell F), Stensland M, Lipchik GL, Cordingley GE, Carlson B. Management of chronic tension-type headache with tricyclic antidepressant medication, stress-management therapy, and their combination: a randomized controlled trial. JAMA 2001;285:2208-15.

55 Lipchik G, Holroyd K, Nash J. Cognitive-behavioral management of recurrent headache disorders: a minimal-therapist contact approach. In: Turk D, Gatchel R, eds. Psychological approaches to pain management. 2nd ed. Guilford Press, 2002:356-89.

56 Holroyd K, Cottrell C, Echelberger-McCune R. Behavioral management for migraine headaches: a treatment program. Ohio University Headache Treatment and Research Project, 2000.

57 Bernstein D, Borkovec T, Hazlett-Stevens H. New directions in progressive relaxation training: a guidebook for helping professions. Praeger Publishers, 2000.

58 Beck A. Cognitive approaches to stress. In: Lehrer P, Woolfolk R, eds. Principles and practice of stress management. Guilford Press, 1993:333-71.

59 Blanchard E, Andrasik F, Neff D, Jurish S, O’Keefe D. Social validation of the headache diary. Beh Ther 1981;12:711-5.

60 Holroyd K, Chen Y. A hand-held computer headache diary program monitoring headaches, medication use, and disability in real time. Cephalalgia 2000;36:123.

61 Martin B, Pathak D, Sharfman M, Adelman J, Taylor F, Kwong W, et al. Validity and reliability of the migraine-specific quality of life questionnaire (MSQL version 2.1). Headache 2000;40:204-15.

62 Patrick D, Hurst B, Hughes J. Further development and testing of the migraine-specific quality of life (MSQL) measure. Headache 2000;40:550-60.

63 Blanchard E, Schwarz S. Clinically significant changes in behavioral medicine. Behav Assess 1988;10:171-88.

64 McCulloch C, Searle S. Generalized linear and mixed models. John Wiley \& Sons, 2000

65 Mallinckrodt C, Clark W, David S. Accounting for dropout bias using mixed-effects models. J Biopharm Stat 2001;11:9-21.

66 Lane P. Handling drop-out in longitudinal clinical trials: a comparison of the LOCF and MMRM approaches. Pharm Stat 2008;7:93-106.

67 Molenberghs G, Thijs H, Jansen I, Beunckens C. Analyzing incomplete longitudinal clinical trial data. Biostatistics 2004;5:445-64.

68 Mallinckrodt C, Watkin J, Molenberghs G, Carroll R. Choice of the primary analysis in longitudinal clinical trials. Pharm Stat 2004;3:161-9.

69 Mallinckrodt C, Sanger T, Dube S, DeBrota D, Molenberghs G, Carroll R, et al. Assessing treatment effects in longitudinal data. Biol Psychiatry 2003;53:754-60.

70 Barnes S, Mallinckrodt C, Lindborg S, Carter M. The impact of missing data and how it is handled on the rate of false-positive results in drug development. Pharm Stat 2008;7:215-25.

71 Lipton RB, Stewart WS, Simon D. Medical consultation for migraine: results from the American Migraine Study. Headache 1998;38:87-96

72 Dasilva AF, Granziera C, Snyder J, Hadjikhani N. Thickening in the somatosensory cortex of patients with migraine. Neurology 2007;69:1990-5.

73 Kruit M. Brain stem and cerebellar hyperintense lesions in migraine. Stroke 2006;37:1109-12

74 Hro'bjartsson A, Gotzsche P. Is the placebo powerless? Update of a systematic review with 52 new randomized trials comparing placebo with no treatment. J Intern Med 2004;256:91-100. 
75 Mathew N, Kailsam J, Gentry P, Chernyshev O. Treatment of nonresponders to oral sumatriptan with zolmitriptan and rizatriptan a comparative open trial. Headache 2000;40:464-5.

76 Silberstein S, Dodick D, Kesslick J. Removing barriers to appropriate migraine treatment: formulary limitations and triptan package size. Headache 2005;45:1250-4.

77 Lipton R, Bigal M. The epidemiology and impact of migraine. In Lipton R, Bigal M, eds. Migraine and other headache disorders. Taylor \& Francis Group, 2006:23-36.

78 Ziegler D, Hurwizt A, Preskorn S, Hassanein R, Seim J. Propranolol and amitriptyline in prophylaxis of migraine. Arch Neurol 1993;50:825-30.

79 Zieglar D, Hurwitz A, Hassanein R, Kodanaz H, Preskorn S, Mason J. Migraine prophylaxis: a comparison of propranolol and amitriptyline. Arch Neurol 1987;44:486-9.

80 Solomon G. Verapamil and propranolol in migraine prophylaxis: a double-blind, crossover study. Headache 1986;20:325.

81 Kaniecki R. A comparison of divalproex with propranolol and placebo for the prophylaxis of migraine without aura. Arch Neurol 1997;54:1141-5.

82 Kangasniemi T, Nyrke T, Lang AH, Petersen E. Femoxetine-a new 5 HT uptake inhibitor and propranolol in the prophylactic treatment of migraine. Acta Neurol Scand 1983;68:262-7.

83 Dahlof C. Flunarizine vs long acting propranolol in the prophylactic treatment of migraine: a double-blind parallel group study. In: Rose F, ed. New advances in headache research. Smith-Gordon, 1989:281-9.
84 Diener $\mathrm{H}$, Foh $\mathrm{M}$, laccarino $\mathrm{C}$, Wessely $\mathrm{P}$, Isler $\mathrm{H}$, Strenge $\mathrm{H}$, et al. Cyclandelate in the prophylaxis of migraine: a randomized, parallel, double-blind study in comparison with placebo and propranolol. Cephalalgia 1996;16:441-7.

85 Diener H, Matias-Guiu J, Hartung E, Pfaffenrath V, Ludin H, Nappi G, et al. Efficacy and tolerability in migraine prophylaxis of flunarizine in reduced doses: a comparison with propranolol $160 \mathrm{mg}$ daily. Cephalalgia 2002;22:209-21.

86 Gawel J, Kreeft J, Nelson R, Simard D, Arnott W. Comparison of the efficacy and safety of flunarizine to propranolol in the prophylaxis of migraine. Can J Neurol Sci 1992;19:340-5.

87 Johnson R, Hornabrook R, Labie D. Comparison of mefenamic acid and propranolol with placebo in migraine prophylaxis. Acta Neurol Scand 1986;73:490-2.

88 Mikkelson B, Kjaersgaard K, Christiansen L. Prophylactic treatment of migraine with tolfenamic acid, propranolol and placebo. Acta Neurol Scand 1986;73:423-7.

89 Olerud B, Gustavsson C, Furberg B. Nadolol and propranolol in migraine management. Headache 1986;26:490-3.

90 Rao B, Das D, Taraknath V, Sarma Y. A double blind controlled study of propranolol and cyproheptadine in migraine prophylaxis. Neurol India 2000;48:223-6.

91 Blanchard E. Psychological treatment of benign headache disorders. J Consult Clin Psychol 1992;60:537-51.

Accepted: 29 June 2010 